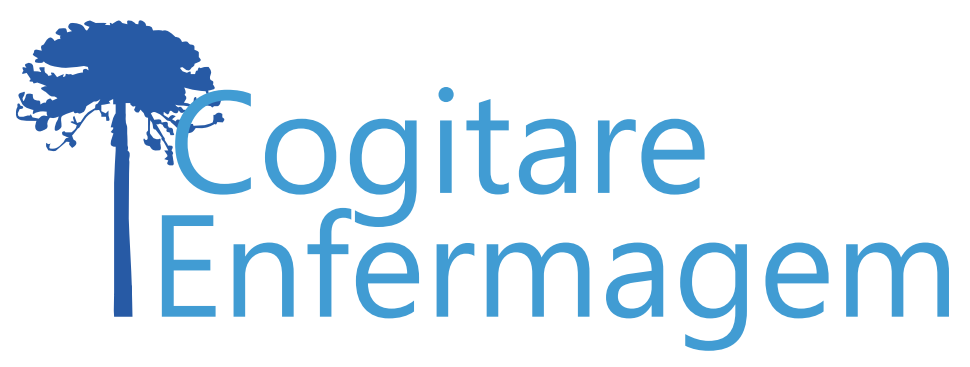

ARTIGO ORIGINAL

\title{
FERRAMENTA DE AVALIAÇÃO DA QUALIDADE: MAPEAMENTO DE SINAIS CLÍNICOS DE MORTE ENCEFÁLICA
}

\author{
Neide da Silva Knihs ${ }^{1}$ \\ Aline Lima Pestana Magalhães ${ }^{1}$ \\ Michele Cristina Pires Semeão ${ }^{1}$ \\ Rosane Lucilene dos Santos ${ }^{1}$ (1) \\ Gabriela Marcellino de Melo Lanzoni ${ }^{1}$ \\ Daniela Couto Carvalho Barra ${ }^{1}$ (1)
}

\section{RESUMO}

Objetivo: identificar características dos óbitos e sinais clínicos de morte encefálica dos pacientes com lesão neurológica internados em unidades de pacientes críticos, a partir de ferramenta para apoio na busca ativa.

Método: estudo descritivo, retrospectivo, realizado de janeiro a agosto de 2017 nas unidades críticas de dois hospitais do sul brasileiro. Os dados foram coletados nos prontuários de pacientes que evoluíram a óbito utilizando-se instrumento da Organização Nacional de Transplantes da Espanha, analisados pela estatística descritiva.

Resultados: os sinais clínicos de morte encefálica identificados antes do óbito foram Glasgow três; reflexos de tosse ausente; hipertensão intracraniana presente, pupilas midriáticas e poliúria. Destacase que $14,8 \%$ apresentaram todos os critérios para iniciar o diagnóstico de morte encefálica, mas não foram notificados à Central Estadual de Transplante.

Conclusão: os profissionais atuantes nessas unidades devem ter conhecimentos dos sinais clínicos de morte encefálica para que ocorra identificação precoce desses pacientes, consequentemente, aumentando as notificações.

DESCRITORES: Morte Encefálica; Perfil de Saúde; Gestão da Qualidade; Obtenção de Tecidos e Órgãos; Enfermagem.

\section{HERRAMIENTA DE LA EVALUACIÓN DE LA CALIDAD: MAPEO DE LOS SIGNOS CLÍNICOS DE LA MUERTE CEREBRAL}

\section{RESUMEN:}

Objetivo: identificar las características de las muertes y los signos clínicos de muerte cerebral de los pacientes con lesión neurológica ingresados en unidades de pacientes críticos, a partir de una herramienta de apoyo a la búsqueda activa. Método: estudio descriptivo y retrospectivo realizado de enero a agosto de 2017 en las unidades críticas de dos hospitales del sur de Brasil. Los datos se recogieron en los prontuarios de pacientes que evolucionaron al óbito utilizando el instrumento de la Organización Nacional de Trasplantes de España, analizados por la estadística descriptiva. Resultados: os sinais clínicos de morte encefálica identificados antes do óbito foram Glasgow três; reflexos de tosse ausente; hipertensão intracraniana presente, pupilas midriáticas e poliúria. Destaca-se que 14,8\% apresentaram todos os critérios para iniciar o diagnóstico de morte encefálica, mas não foram notificados à Central Estadual de Transplante. Resultados: los síntomas clínicos de muerte encefálica identificados antes del óbito fueron Glasgow tres; reflejos de tos ausentes; hipertensión intracraneal presente, pupilas midriáticas y poliuria. Cabe destacar que el 14,8\% presentaba todos los criterios para iniciar el diagnóstico de muerte cerebral, pero no fueron notificados al Centro Estatal de Trasplantes. Conclusión: los profesionales que trabajan en estas unidades deben tener conocimiento de los signos clínicos de la muerte cerebral para la identificación temprana de estos pacientes, aumentando así las notificaciones.

DESCRIPTORES: Muerte Encefálica; Perfil de Salud; Gestión de la Calidad; Obtención de Tejidos y Órganos. 
A Morte Encefálica (ME) vem sendo estudada ao longo dos anos sob diferentes aspectos devido às alterações fisiológicas causadas por essa condição(1). A compreensão dos profissionais de saúde para atuar junto a esses pacientes, aliada ao desenvolvimento de cuidados efetivos, agilizam o diagnóstico e notificação da ME à Central Estadual de Transplante $(C E T)^{(1-2)}$.

Considerando os diversos aspectos estudados sobre a temática da $\mathrm{ME}$, questionase: existe a necessidade de investigar questões relacionadas à ME? É possível pensar, brevemente, que não. Contudo, os dados da Associação Brasileira de Transplante de Órgãos (ABTO) de 2019 evidenciaram subnotificação de aproximadamente 3.800 MEs por ano às CETs no Brasil(3). Observa-se realidade semelhante em estudo brasileiro desenvolvido em três hospitais de referência em neurologia, que evidenciou que $25 \%$ das MEs não foram identificadas e notificadas às CETs ${ }^{(4)}$.

Além da subnotificação da $M E$, existem dificuldades das equipes que atuam em Unidades de Pacientes Críticos (UCls) quanto à identificação, avaliação e validação de pacientes com critérios clínicos para iniciar e executar o diagnóstico de $\mathrm{ME}^{(5-7)}$, que reforçam a importância da temática.

Vale ressaltar que existem controvérsias éticas relacionadas ao desconhecimento e diferenciação dos conceitos de morte por parada cardíaca, conceito de ME e manutenção hemodinâmica do paciente em ME. Isso acontece devido a este paciente permanecer com as funções fisiológicas preservadas, mesmo que temporariamente. Além disso, a equipe depara-se com questões culturais, religiosas e éticas ao manter artificialmente um paciente sem função cerebral ${ }^{(8-9)}$.

Neste contexto, estão envolvidas as questões legais de iniciar os procedimentos para diagnóstico de $\mathrm{ME}$ em todos os pacientes que apresentam coma não perceptivo e apneia, independente da condição de doador ou não. A partir da Lei $n^{\circ}$ 9434/1997, foi estabelecido que a notificação de ME às CETs seria compulsória(1-2). Esta ação é primordial, pois a efetividade da doação de órgãos e tecidos está diretamente relacionada à precocidade da notificação para se evitar a perda do potencial doador.

Para facilitar a identificação dos pacientes com critérios clínicos de $\mathrm{ME}$, a Portaria $n^{\circ} 2.600 / 2009$ aponta como obrigatória a busca ativa, in loco, em unidades de terapias intensivas, emergências e demais unidades de internação que cuidam desses pacientes. As visitas nessas unidades devem ser realizadas diariamente pelos profissionais das Comissões Hospitalar de Transplante $(\mathrm{CHT})^{(10-11)}$, que, em sua maioria, é composta por enfermeiros.

A Resolução do Conselho Federal de Medicina destaca o compromisso ético e legal que a equipe de saúde, em especial médicos, devem ter na identificação dos pacientes com critérios clínicos de ME, para que este diagnóstico seja iniciado o mais breve possível (2). Destaca-se que a avaliação neurológica do paciente com potencial evolução para ME tem amparo por diversos critérios clínicos ${ }^{(12-13)}$.

Salienta-se que a escassez de órgãos é um problema mundial e a elaboração de estratégias para conhecer o número de MEs, das subnotificações e das causas de perdas de possíveis doadores são essenciais para aprimorar o processo de doação-transplantes ${ }^{(4,14)}$. Além disso, a manutenção desse paciente deve ser adequada, para que não haja sua perda antes da família ser consultada sobre a doação de órgãos ${ }^{(15)}$.

Assim, compreende-se como relevante que a equipe de saúde conheça os principais critérios clínicos de $M E$, em especial o enfermeiro e sua equipe, pois estão envolvidos 24 horas no cuidado ao paciente nas UCls, acompanhando todas as mudanças do prognóstico de um paciente neurológico crítico(1). 
O presente estudo fez uso do instrumento do Programa de Garantia de Qualidade (PGQ) no processo de doação de órgãos e transplantes da Espanha. A escolha do instrumento para aplicação no contexto brasileiro deu-se pelo destaque que Espanha ganhou no processo de doação de órgãos, saindo de uma taxa de 14,3 notificações de potenciais doadores (pmp) em 1989 para 48,9 pmp em 2019, tornando-se uma referência mundial em doação de órgãos e transplantes ${ }^{(16)}$.

Mediante o exposto, o objetivo do estudo é identificar características dos óbitos e sinais clínicos de morte encefálica dos pacientes com lesão neurológica internados em unidades de pacientes críticos, a partir de ferramenta para apoio na busca ativa.

\section{MÉTODO}

Trata-se de um estudo quantitativo, retrospectivo, descritivo, desenvolvido nas unidades de pacientes críticos (UCl), serviço de emergência e Unidade de Terapia Intensiva (UTI) de dois hospitais de grande porte em neurologia no sul do país, sendo denominados Hospital 1 e Hospital 2. A escolha das duas instituições ocorreu devido à elevada demanda de pacientes neurocirúrgicos, pelo quantitativo de leitos de UTI, o número de notificações de possíveis doadores à Central Estadual de Transplantes e principalmente pelo número de perdas de possíveis doadores ${ }^{(17)}$.

A primeira instituição é referência em neurocirurgia, com 260 leitos, sendo 20 leitos de UTI. A CHT é composta por cinco enfermeiros e um médico. A busca ativa é realizada por essa equipe, diariamente, em três horários, nas unidades de pacientes críticos que apresentam pacientes com lesão neurológica, utilizando o instrumento da Portaria $n^{\circ}$ 2.600/2009 adaptado pela CET-SC, checando todas as variáveis do instrumento (causa da lesão neurológica, pupila, Escala Glasgow, sedação sim ou não, presença de poliúria, sim ou não; PIC sim ou não. Qual o Valor?). Em 2017, foram notificados 52 potenciais doadores para a CET, e 27 tornaram-se doadores efetivos.

A segunda instituição localiza-se na região Norte de Santa Catarina, servindo como referência no atendimento em Urgência e Emergência adulto, alta complexidade em neurocirurgia, ortopedia e traumatologia, oncologia e transplantes. Apresenta 255 leitos, sendo 20 leitos de UTI. Em 2017, 43 pacientes foram notificados à CET como possíveis doadores e 24 foram doadores efetivos ${ }^{(17)}$. A equipe da $\mathrm{CHT}$ é composta por cinco enfermeiros e dois médicos, que realizam a busca ativa diariamente, em três horários, utilizando o instrumento supracitado.

A coleta dos dados ocorreu em prontuários de pacientes que foram a óbito nas referidas UCls entre janeiro a agosto de 2017 no Hospital 1 e de fevereiro a julho de 2017 no Hospital 2. Todos os prontuários de pacientes que foram a óbito no período preestabelecido integraram o estudo. No entanto, excluiu-se aqueles com informações incompreensíveis. A justificativa do recorte temporal ocorreu devido ter iniciado nesse período a implantação do uso das ferramentas de qualidade no processo de doação de órgãos e tecidos em Santa Catarina.

A coleta das informações ocorreu por meio de um dos instrumentos do PGQ no processo de doação de órgãos e transplantes da Espanha. Os instrumentos do PGQ foram elaborados por profissionais da Organização Nacional de Transplantes (ONT), e foram traduzidos e adaptados para o Brasil(18). Obteve-se autorização prévia para a utilização do instrumento.

O PGQ é formado por três instrumentos. Neste estudo, utilizou-se o instrumento intitulado "Informativo do óbito", que trata da caracterização do paciente que evoluiu para ME e tem como objetivos: definir a capacidade de doação de órgão conforme o tipo 
de hospital; avaliar se os prontuários eram de pacientes com critérios clínicos para iniciar o protocolo de $M E$, ou ainda, representava um prontuário de um paciente que teve o diagnóstico de $\mathrm{ME}$, mas não foi notificado à CET.

Na primeira etapa do instrumento, coleta-se informações da primeira evolução da equipe multiprofissional de saúde, identificando: Idade; Sexo; se há presença de lesão neurológica e qual a causa da lesão neurológica; Glasgow de entrada (levanta-se respostas referentes à abertura ocular, melhor resposta verbal e melhor resposta motora, podendo a pontuação variar entre 3 e 15, com um escore inferior a 8 indicando uma lesão grave, 9 a 12 lesão moderada e 15, lesão mínima ${ }^{(19)}$. Instalada a monitorização da Pressão Intracraniana (PIC)? Se sim, qual o valor da PIC? Característica da pupila; Presença de reflexo de tosse e de poliúria. Utilizou-se na pesquisa a versão antiga da Escala de Coma de Glasgow.

$\mathrm{Na}$ segunda etapa do instrumento, busca-se informações nas últimas evoluções da equipe de saúde antes da morte do paciente. Nesse momento, os dados envolvem: Se estava sem sedação e há quanto tempo; o último Glasgow; o valor da PIC; característica da pupila; presença de reflexo de tosse e de poliúria; o diagnóstico de ME havia sido iniciado. Caso seja confirmada uma possível ME, esse instrumento permite identificar as causas da não abertura do protocolo de ME e as causas de não notificação da ME à CET. Contudo, nesse estudo, a proposta foi identificar os sinais clínicos que indiquem uma possível ME.

$O$ preenchimento desse instrumento foi realizado pelos enfermeiros das $\mathrm{CHT}$, previamente capacitados, com tempo médio de 10 minutos. Os dados foram organizados em um banco de dados do programa Excel® 2010, e procedeu-se à análise estatística descritiva simples.

Destaca-se que o presente estudo está vinculado ao macroprojeto intitulado: "Ferramentas de gestão para analisar as causas de perda de possíveis e potenciais doadores de órgãos e tecidos" e obteve aprovação pelo Comitê de Ética em Pesquisa, sob parecer número 1.410 .164 .

\section{RESULTADOS}

Os resultados serão apresentados em duas etapas: primeiramente, a caracterização dos óbitos ocorridos no Hospital 1 e 2 em relação ao sexo dos pacientes e condição clínica/motivo da internação. E na sequência, os sinais clínicos de ME dos pacientes com lesão neurológica.

Foram identificados 504 prontuários de pacientes que evoluíram para morte. No Hospital 1, foram encontrados 303 prontuários, sendo que 175 (57,8\%) eram homens, $124(41 \%)$ mulheres e em quatro $(1,2 \%)$ prontuários não houve registro quanto ao sexo do paciente. No Hospital 2 foram encontrados 201 prontuários, sendo que $118(58,7 \%)$ casos eram homens e $83(41,3 \%)$ mulheres.

Quanto aos motivos da internação, no Hospital 1, 88 (29\%) foram por causas neurológicas, $33(10,9 \%)$ por causas cardíacas, $26(8,6 \%)$ por choques e $156(51,5 \%)$ distribuídos por outras causas (renais, câncer, respiratórias, parada cardíaca, digestivas, hepáticas, traumas, vasculares, endócrinas, queimadura, HIV e causas desconhecidas). No Hospital 2, foram $36(17,9 \%)$ por causas respiratórias, 33 (16,4\%) por causas neurológicas, $24(11,9 \%)$ por causas renais e $108(53,8 \%)$ distribuídos em outras causas. Dos 121 pacientes que internaram por causas neurológicas, $36(29,7 \%)$ foram por traumatismo craniano, $36(29,7 \%)$ acidente vascular hemorrágico, $21(17,3 \%)$ acidente vascular isquêmico e 28 $(23,14 \%)$ por outras causas (Tabela 1$)$. 
Tabela 1 - Causas de internação no Hospital 1 e Hospital 2. Florianópolis, SC, Brasil, 2017

\begin{tabular}{|c|c|c|c|c|c|}
\hline \multicolumn{2}{|c|}{ Causas de internação } & \multicolumn{2}{|c|}{ Hospital 1} & \multicolumn{2}{|c|}{ Hospital 2} \\
\hline & & $\mathbf{N}$ & $\%$ & $\mathbf{n}$ & $\%$ \\
\hline \multirow{5}{*}{$\begin{array}{l}\text { Causas } \\
\text { neurológicas }\end{array}$} & Traumatismo de crânio & 36 & 11,9 & 4 & 2 \\
\hline & Acidente vascular hemorrágico & 29 & 9,5 & 13 & 6,4 \\
\hline & Acidente vascular isquêmico & 14 & 4,6 & 7 & 3,5 \\
\hline & Crise convulsiva & 0 & 0 & 4 & 2 \\
\hline & Outros & 9 & 3 & 5 & 2,5 \\
\hline \multirow{11}{*}{$\begin{array}{l}\text { Outras } \\
\text { causas }\end{array}$} & Causas cardíacas & 33 & 10,9 & 16 & 8 \\
\hline & Choques & 26 & 8,6 & 7 & 3,5 \\
\hline & Causas renais & 24 & 7,9 & 24 & 11,9 \\
\hline & Câncer & 18 & 5,9 & 15 & 7,5 \\
\hline & Causas respiratórias & 16 & 5,3 & 36 & 17,9 \\
\hline & Parada cardíaca & 15 & 4,9 & 9 & 4,5 \\
\hline & Causas digestivas & 14 & 4,6 & 0 & 0 \\
\hline & Causas hepáticas & 12 & 4 & 12 & 6 \\
\hline & Traumas & 10 & 3,3 & 5 & 2,5 \\
\hline & Sem registro & 0 & 0 & 9 & 4,5 \\
\hline & Outras & 47 & 15,6 & 35 & 17,5 \\
\hline \multicolumn{2}{|c|}{ Total de causas neurológicas } & 88 & 29 & 33 & 16,4 \\
\hline \multicolumn{2}{|c|}{ Total de outras causas } & 215 & 71 & 168 & 83,6 \\
\hline \multicolumn{2}{|l|}{ TOTAL } & 303 & 100 & 201 & 100 \\
\hline
\end{tabular}

Fonte: Autores (2017).

Dos pacientes com lesão neurológica no momento da hospitalização, 37 (31\%) foram hospitalizados com Glasgow três; 28 (23\%) apresentavam pupilas anisocóricas e oito (7\%) estavam com midríase bilateral, $32(26,4 \%)$ reflexos de tosse ausente, e $26(21,5 \%)$ presença de hipertensão intracraniana. Nenhum dos pacientes apresentou poliúria no momento da internação (Figura 1). 


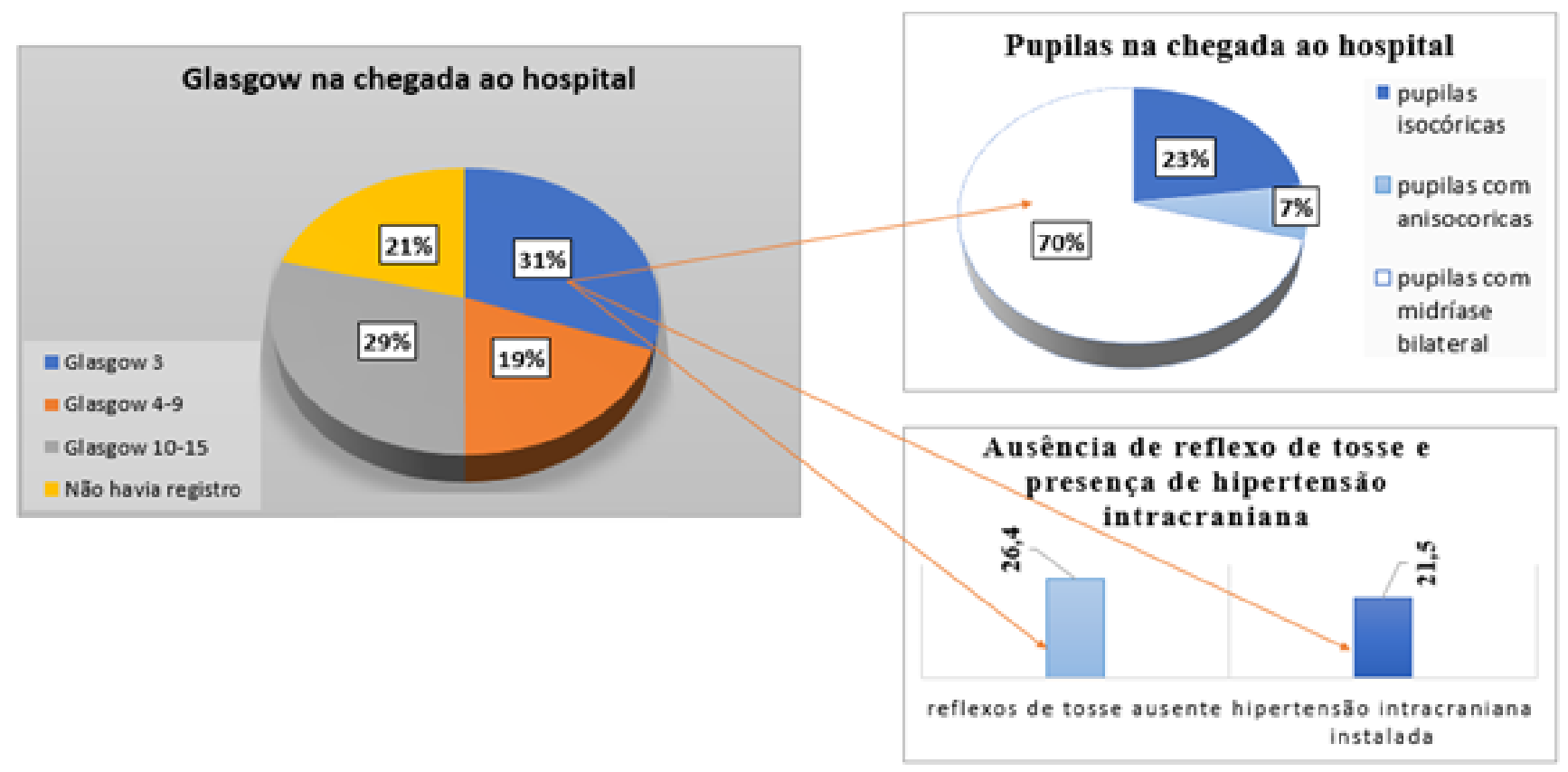

Figura 1 - Características dos pacientes com lesão neurológica no momento da internação nos hospitais. Florianópolis, SC, Brasil, 2017

Fonte: Autores (2017).

A média de tempo de internação entre a chegada ao hospital até a data do óbito em pacientes com lesão neurológica foi de quatro dias. No que se refere às informações obtidas nos prontuários, as quais representam sinais clínicos de ME antes do óbito, 75 (62\%) apresentaram Glasgow três, cinco (4\%) Glasgow entre quatro e nove, seis (5\%) com Glasgow 10-15 e em 12 (10\%) não havia registro de Glasgow; 23 (19\%) pacientes estavam sedados no momento do óbito (Figura 2).

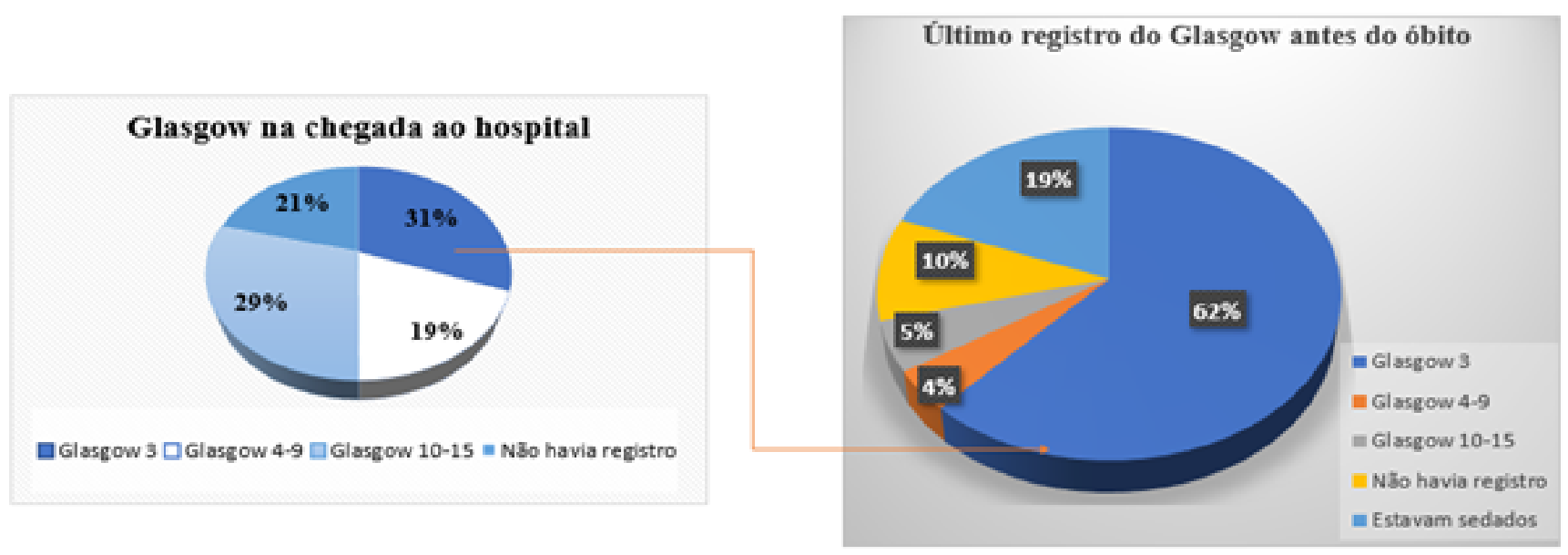

Figura 2 - Comparação entre o Glasgow de chegada com o Glasgow antes do óbito. Florianópolis, SC, Brasil, 2017

Fonte: Autores (2017). 
No que se refere aos outros sinais clínicos de ME, 63 (52\%) estavam com midríase bilateral, $61(50,4 \%)$ reflexo de tosse ausente, 88 (72,7\%) presença de hipertensão intracraniana e $102(84,3 \%)$ poliúria (Figura 3).

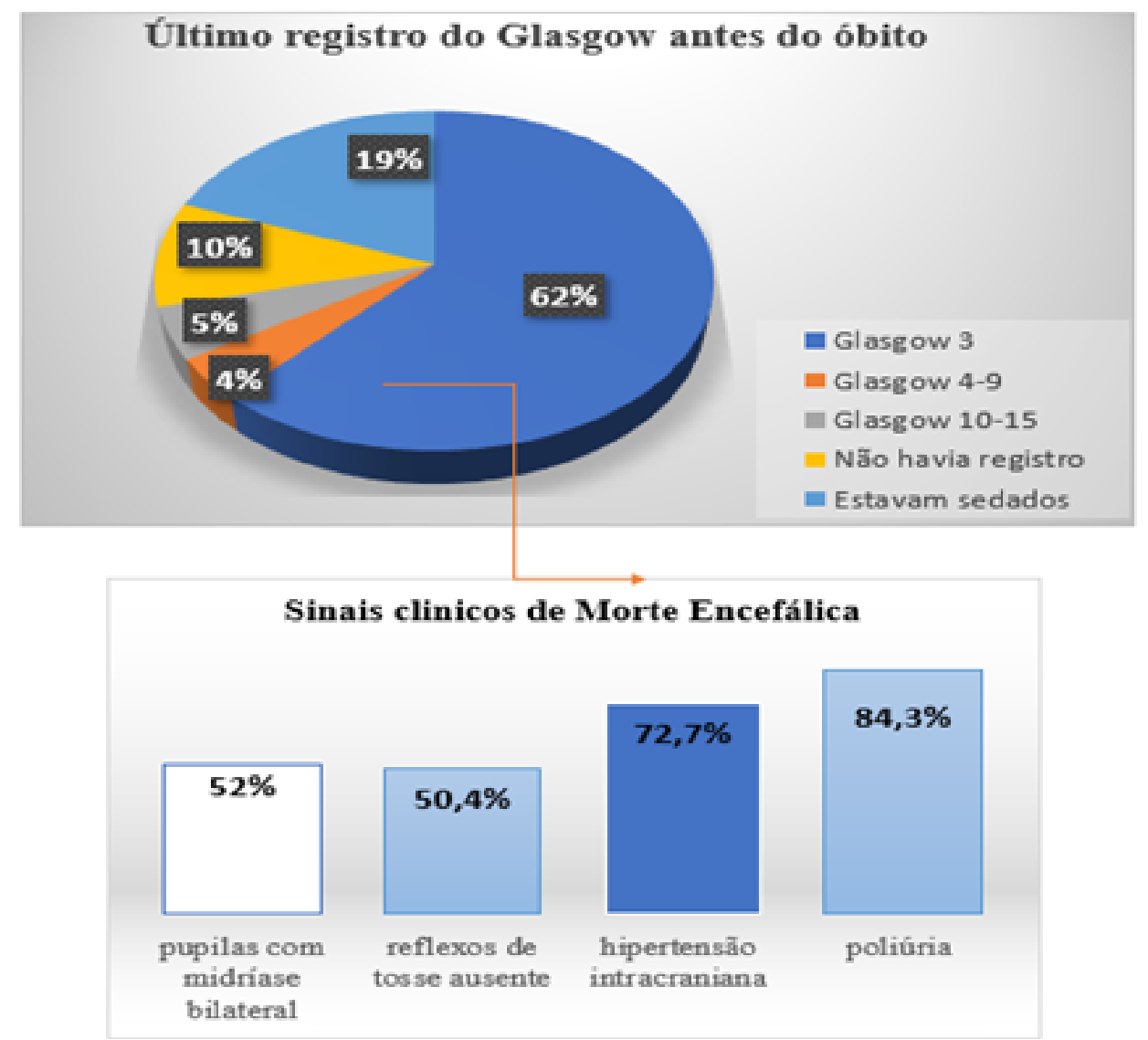

Figura 3 - Distribuição dos sinais clínicos de morte encefálica identificados nos prontuários. Florianópolis, SC, Brasil, 2017

Fonte: Autores (2017).

Das mortes por causas neurológicas nos dois hospitais (121), foram identificados 34 $(28,1 \%)$ pacientes com ME que foram notificados à CET, $23(19 \%)$ pacientes apresentaram um ou mais critérios para evoluir a ME e $18(14,8 \%)$ apresentaram todos os critérios para iniciar o diagnóstico de ME, mas não foram notificados à CET.

\section{DISCUSSÃO}

Identificar sinais clínicos de ME ainda é um grande desafio para os profissionais de saúde, por terem sido preparados na graduação para salvar vidas. Estar diante de um paciente com possibilidade de iniciar o diagnóstico de ME traz reflexões éticas, morais, legais, pessoais, culturais e religiosas. Há profissionais que concordam que a ME é equivalente à morte, outros que a morte só ocorre diante da parada cardíaca (20). Para enfermeiros, há incerteza quanto a este diagnóstico, mas entendem que o cuidado a esse 
paciente em ME faz parte da rotina das UCls e que o mesmo pode ajudar outras pessoas por meio da doação de órgãos e tecidos ${ }^{(20-21)}$.

Nessa perspectiva, compreende-se a importância de a equipe de saúde ter conhecimento, habilidade e atitude para reconhecer precocemente pacientes com critérios clínicos de ME. Assim, entende-se que o estudo mostra informações essenciais sobre os critérios clínicos para iniciar o diagnóstico de ME que servirão de apoio aos profissionais das UCls e CHT na busca efetiva desses pacientes.

Evidenciou-se no estudo que o maior índice de causas neurológicas está relacionado a homens por Traumatismo cranioencefálico (TCE), seguido de AVC hemorrágico. Grande parte desses pacientes, 31\%, chegam ao hospital com Glasgow três, 23\% pupilas anisocóricas, $5 \%$ reflexo de tosse ausente e 21,5\% hipertensão intracraniana. Outros estudos corroboram com esses resultados quanto ao perfil do doente neurocrítico: homem, vítima de TCE, além de apresentarem prognóstico ruim na chegada ao hospital e Glasgow inferior a quatro pontos ${ }^{(12,22-23)}$. Destaca-se ainda que doentes neurocríticos com Glasgow inferior a quatro e hipertensão intracraniana não controlada tem forte probabilidade de evoluir para $\mathrm{ME}^{(24)}$. Em estudo realizado na Itália com 569 pacientes, 7\% apresentaram Glasgow três, tendo piora ao longo da internação e evoluindo para $\mathrm{ME}^{(25)}$.

Nesse estudo, os dados comprovam essa gravidade do doente neurocrítico, em razão de $30 \%$ chegarem com Glasgow três ao hospital, 62\% apresentarem Glasgow três antes do óbito, hipertensão intracraniana e ausência de reflexos de tronco. Com a piora progressiva durante a internação, aumenta a demanda de cuidados relacionada à instabilidade hemodinâmica ocasionada pela lesão neurológica grave. Estudos apontam que doentes neurocríticos demandam cuidados intensivos, em especial quando a PIC é instalada, a qual normatiza fluxo sanguíneo cerebral e sua perfusão. Em caso de não controle da PIC, esse doente poderá evoluir, rapidamente, para $\mathrm{ME}^{(22)}$.

Tais informações corroboram com os achados deste estudo, onde acima de $70 \%$ dos pacientes apresentavam hipertensão intracraniana, acima de $80 \%$ apresentavam poliúria, mais de $50 \%$ estavam com reflexo de tosse ausente e pupilas midriáticas, indicando sinais clínicos de lesão neurológica grave. Contudo, os dados do estudo evidenciam a subnotificação de potenciais doadores à CET, corroborando com os dados apresentados pela ABTO, estimando que, aproximadamente, 3800 a 5000 pacientes com sinais clínicos de ME deixam de ser identificados e notificados anualmente ${ }^{(3)}$. A subnotificação representa uma das principais causas da não efetividade da doação de órgãos e tecidos de doador falecido(4,14).

A legislação vigente no Brasil determina que todos os pacientes considerados possíveis doadores de órgãos e tecidos, os quais estão em coma aperceptivo, devem ser identificados e mapeados para serem acompanhados até que haja melhora clínica, evolução para diagnóstico de ME ou parada cardíaca, evitando, assim, a perda de possíveis doadores $^{(11)}$.

As perdas de possíveis doadores (pacientes com critérios clínicos de ME) estão diretamente relacionadas à instabilidade hemodinâmica envolvendo a hipotensão, poliúria, sódio superior a $155 \mathrm{mEq} / \mathrm{l}$ e temperatura inferior a $35,5^{\circ} \mathrm{C}^{(4,14,26)}$. Para minimizar essas perdas, diretrizes e programa de qualidade orientam a busca ativa efetiva em todas as unidades em que estão internados doentes neurocríticos ${ }^{(12,27-28)}$. Contudo, muitas vezes pela falta ou pouca capacitação de como desenvolver a busca ativa e quais critérios avaliar, o enfermeiro acaba não rastreando todos os pacientes com alterações neurológicas graves que indicam a possibilidade de iniciar o diagnóstico de $\mathrm{ME}^{(29)}$.

Assim, compreende-se que os dados obtidos nesse estudo permitem mapear sinais clínicos de ME por meio da busca ativa, bem como gerenciar o cuidado imediato de quaisquer alterações hemodinâmicas que possam surgir diante da lesão neurológica, minimizando risco de parada cardíaca deste possível doador, além de viabilizar o diagnóstico de ME o mais breve possível. 
A participação do enfermeiro na busca ativa, identificação, validação, notificação e manutenção do potencial doador tem contribuído de maneira efetiva no processo de doação de órgãos e tecidos para fins de transplante ${ }^{(29-30)}$. Destaca-se que a busca ativa em possíveis doadores é uma responsabilidade da CHT/CIHDOTT, em especial dos enfermeiros por serem os profissionais em maior número integrantes desta comissão ${ }^{(1,30)}$.

Salienta-se que a incompreensão dos sinais clínicos de ME por parte dos profissionais que atuam nas UCls e CHT/CIHDOTT pode repercutir na qualidade dos órgãos e tecidos a serem transplantados, podendo até mesmo levar à perda do possível doador.

As limitações do estudo relacionam-se a ser aplicado a contextos específicos, circunscrito a dois hospitais de um estado brasileiro. Assim, sugere-se que novas pesquisas sejam implementadas em diferentes regionalidades, proporcionando comparação dos achados e mapeando os sinais clínicos e as subnotificações da ME no país.

\section{CONCLUSÃO}

Este estudo atingiu o objetivo ao identificar os sinais clínicos dos pacientes que evoluíram para ME, a partir de um instrumento do programa de garantia de qualidade do processo de doação de órgãos e transplante da Espanha, como uma alternativa à otimização da busca ativa ao paciente neurológico.

Destaca-se a importância dos profissionais atuantes nas unidades de pacientes críticos terem conhecimentos desses sinais clínicos de $M E$, para que se otimize a identificação e a operacionalização do protocolo de diagnóstico de $\mathrm{ME}$, de forma a garantir um maior número de captação e transplantes de órgãos.

Acredita-se que os resultados desse estudo possibilitarão aos gestores a elaboração de estratégias, como a capacitação dos profissionais de saúde, para a identificação precoce dos pacientes nessas condições, de modo a contribuir com o aumento no número de notificações à CET.

\section{REFERÊNCIAS}

1. Brasil. Presidência da República. Casa Civil. Subchefia para Assuntos Jurídicos. Decreto n. 9175, de 18 de outubro de 2017: regulamenta a Lei n. 9.434, de 4 de fevereiro de 1997, para tratar da disposição de órgãos, tecidos, células e partes do corpo humano para fins de transplante e tratamento. Casa Civil. [Internet]. Brasília (DF): 2017. [acesso em 10 fev 2020]. Disponível em: http://www.planalto.gov.br/ ccivil 03/ Ato2015-2018/2017/Decreto/D9175.htm.

2. Conselho Federal de Medicina. Resolução CFM n. 2173, de 23 novembro 2017. Define os critérios do diagnóstico de morte encefálica [Internet]. Brasília (DF): CFM; 2017. [acesso em 10 jul 2019]. Disponível em: https://sistemas.cfm.org.br/normas/visualizar/resolucoes/BR/2017/2173.

3. Registro Brasileiro de Transplante (RBT). Associação Brasileira de Transplantes de Órgãos (ABTO). Dimensionamento dos Transplantes no Brasil e em cada estado. [Internet]. 2012-2019. [acesso em $10 \mathrm{mar}$ 2020]. Disponível em: https://www.abto.org.br.

4. Knihs N da S, Magalhães ALP, Santos J, Wolter I dos S, Paim SMS. Doação de órgão e tecidos: utilização de ferramenta de qualidade para a otimização do processo. Esc. Anna Nery. [Internet]. 2019 [acesso em 30 abr 2020]; 23(4). Disponível em: https://doi.org/10.1590/2177-9465-ean-2019-0084. 
5. Cunha DSP, Lira JAC, Ribeiro JF, Campelo GV de S, Silva FAA da, Nunes BMVT. Brain death and organ maintenance: knowledge of intensive care professionals. J Nurs UFPE on line. [Internet]. 2018 [acesso em 30 abr 2019]; 12(1). Disponível em: https://doi.org/10.5205/1981-8963-v12i1a25130p51-58-2018.

6. Bedenko RC, Nisihara R, Yokoi DS, Candido V de M, Galina I, Moriguchi RM, et al. Analysis of knowledge of the general population and health professionals on organ donation after cardiac death. Rev Bras Ter Intensiva. [Internet]. 2016 [acesso em 20 fev 2020]; 28(3). Disponível em: http://dx.doi. org/10.5935/0103-507X.20160043.

7. Castelli I, Costa Junior AL. Health professionals and the diagnosis of encephalic death: a review. Rev. Espacios. [Internet]. 2018 [acesso em 20 fev 2020]; 39(7). Disponível em: http://www.revistaespacios.com/ a18v39n07/a18v39n07p06.pdf.

8. Knihs N da S, Roza B de A, Schirmer J, Ferraz AS. Application of spanish quality instruments about organ donation and transplants validated in pilot hospitals in Santa Catarina. J. Bras. Nefrol. [Internet]. 2015 [acesso em 19 mar 2020]; 37(3). Disponível em: http://dx.doi.org/10.5935/0101-2800.20150052.

9. Bonetti CE, Boes AA, Lazzari DD, Busana J de A, Maestri E, Bresolin P. Organ and tissue donation and reasons for its non-realization. J Nurs UFPE on line. [Internet]. 2017 [acesso em 20 fev 2020]; 11(9). Disponível em: https://periodicos.ufpe.br/revistas/revistaenfermagem/article/view/234483.

10. Brasil. Presidência da República. Casa Civil. Subchefia para Assuntos Jurídicos. Lei n. 9.434 , de 4 de fevereiro de 1997. Dispõe sobre a remoção de órgãos, tecidos e partes do corpo humano para fins de transplante e tratamento e dá outras providências Casa Civil [Internet]. Brasília (DF): 1997. [acesso em 10 jul 2019]. Disponível em: http://www.planalto.gov.br/ccivil 03/LEIS/L9434.htm.

11. Ministério da Saúde (BR). Portaria n. 2600, de 21 outubro 2009. Aprova o Regulamento Técnico do Sistema Nacional de Transplantes. [Internet]. Brasília (DF): 2009. [acesso em 10 jul 2019]. Disponível em: http://bvsms.saude.gov.br/bvs/saudelegis/gm/2009/prt2600 2110 2009.html.

12. Sagishima K, Kinoshita Y. Pupil diameter for confirmation of brain death in adult organ donors in Japan. Acute Med Surg. [Internet]. 2016 [acesso em 22 mar 2020]; 4(1). Disponível em: http://dx.doi. org/10.1002/ams2.208.

13. Barros WCT dos S, Dal Sasso GTM, Alvarez AG, Ramos SF, Martins SR. Aplicativo para avaliação do nível de consciência em adultos: produção tecnológica em enfermagem. Cogit. Enferm. [Internet]. 2019 [acesso em 22 mar 2020]; 24. Disponível em: http://dx.doi.org/10.5380/ce.v24i0.60338.

14. Ludwig EF dos SB, Pereira MCA, Martinez YDE, Mendes KDS, Rossaneis MA. Prototype of a computerized scale for the active search for potential organ donors. Rev Latino-Am. Enfermagem. [Internet]. 2017 [acesso em 2020 mar 22]; 25. Disponível em: http://dx.doi.org/10.1590/15188345.1936.2930.

15. Kock K de S, Santana MG, Silva SC da, Andrade SB, Santos EN dos. Perfil epidemiológico, disfunção orgânica e eletrolítica em potenciais doadores de órgãos e tecidos de um hospital do sul do Brasil. Arq Med Hosp Fac Cienc Med Santa Casa São Paulo. [Internet]. 2019 [acesso em 22 fev 2020]; 64(2). Disponível em: https://doi.org/10.26432/1809-3019.2019.64.2.100.

16. Organización Nacional de Trasplantes. Actividad de donación y transplante españa 2019. [Internet]. 2019 [acesso em 22 set 2020]. Disponível em: http://www.ont.es/infesp/Memorias/.

17. Secretaria de Estado da Saúde SC Transplantes (CET). Dados de doação e transplantes em Santa Catarina. [Internet] 2018 [acesso em 22 mar 2020]. Disponível em: http://sctransplantes.saude.sc.gov.br/ index.php/estatisticas/file/186-estatistica-2018.

18. Knihs N da S, Schirmer J, Roza B de A. Cross-cultural translation of quality instruments in the organ donation process. Acta Paul. Enferm. [Internet]. 2014 [acesso em 22 mar 2020]; 27(1). Disponível em: http://dx.doi.org/10.1590/1982-0194201400012.

19. Humbertjean L, Mione G, Fay R, Durin L, Planel S, Lacour JC, et al. Predictive factors of brain death in severe stroke patients identified by organ procurement and transplant coordination in Lorrain, France. 
Transplant International. [Internet]. 2016 [acesso em 22 set 2020]; 29(3). Disponível em: http://dx.doi. org/10.1111/tri.12695.

20. Magalhães ALP, Erdmann AL, Sousa FGM de, Lanzoni GM de M, Silva EL da, Mello ALSF de. Significados do cuidado de enfermagem ao paciente em morte encefálica potencial doador. Rev Gaúcha Enferm. [Internet]. 2018 [acesso em 12 nov 2020]; 39. Disponível em: http://dx.doi.org/10.1590/19831447.2018.2017-0274.

21. Moghaddam HY, Manzari ZS, Heydari A, Mohammadi E, Khaleghi I. The nursing challenges of caring for brain-dead patients: a qualitative study. Nursing and midwifery studies. [Internet] 2018 [acesso em 20 fev 2020]; 7(3). Disponível em: http://www.nmsjournal.com/text.asp?2018/7/3/116/235638.

22. Arruda F de CA de, Calheiros VP, Sales VM, Bione EC de O, Silva NSB da, Souza MA de O. Cuidados de enfermagem na pressão intracraniana. Revista saúde [Internet]. 2017 [acesso em 22 mar 2020]; 11(esp.1). Disponível em: http://revistas.ung.br/index.php/saude/article/view/3163.

23. Siqueira MM, Araujo CA, Roza B de A, Schirmer J. Indicadores de eficiência no processo de doação e transplante de órgãos: revisão sistemática da literatura. Rev Panam Salud Publica. [Internet]. 2016 [acesso em 22 mar 2020]; 40(2). Disponível em: https://www.scielosp.org/pdf/rpsp/2016.v40n2/90-97/pt.

24. Eira CSL da, Barros MIT de, Albuquerque AMP de. Organ donation: the reality of an intensive care unit in Portugal. Rev Bras Ter Intensiva. [Internet]. 2018 [acesso em 22 mar 2020]; 30(2). Disponível em: https://www.scielo.br/scielo.php?script=sci arttext\&pid=S0103-507X2018000200201.

25. Zappa S, Fagoni N, Bertoni M, Selleri C, Venturirni MA, Finazzi P, et al. Determination of Imminent brain death using the full outline of unresponsiveness score and the glasgow coma scale: a prospective, multicenter, pilot feasibility study. J intensive care med. [Internet]. 2017 [acesso em 16 mar 2020]; 5(1). Disponível em: http://dx.doi.org/10.1177/0885066617738714.

26. Istrate MG, Harrison TR, Valero R, Morgan SE, Páez G, Zhou Q, et al. Benefits of Transplant Procurement Management (TPM) specialized training on professional competence development and career evolutions of health care workers in organ donation and transplantation. Exp Clin Transplant [Internet]. 2015 [acesso em 22 mar 2020]; 13(supl1). Disponível em: https://www.ncbi.nlm.nih.gov/ pubmed/25894145.

27. Escudero D, Valentin MO, Escalante JL, Sanmartin A, Perez-Basterrechea M, Gea J de, et al. Intensive care practices in brain death diagnosis and organ donation. Anaesthesia. [Internet]. 2015 [acesso em 22 mar 2020]; 70(10). Disponível em: http://dx.doi.org/10.1111/anae.13065.

28. Westphal GA, Garcia VD, Souza RL de, Franke CA, Vieira KD, Birckholz VRZ, et al. Guidelines for the assessment and acceptance of potential brain-dead organ donors. Rev Bras Ter Intensiva. [Internet]. 2016 [acesso em 22 mar 2020]; 28(3). Disponível em: https://www.scielo.br/scielo.php?pid=S0103$\underline{507 X 2016000300220 \& s c r i p t=s c i}$ arttext\&tlng=en.

29. Tolfo F, Camponogara S, Montesinos MJL, Siqueira HCH, Scarton J, Beck CLC. A inserção do enfermeiro em comissão intra-hospitalar de doação de órgãos e tecidos. Enfermería Global. [Internet]. 2018 [acesso em 22 mar 2020]; 17(2). Disponível em: https://doi.org/10.6018/eglobal.17.2.289461.

30. Rocha DF da, Canabarro ST, Sudbrack AW. Atribuições de uma organização de procura de órgãos nas atividades da comissão intrahospitalar de doação de órgãos. Rev Bras Promoç Saúde. [Internet]. 2016 [acesso em 22 mar 2020]; 29(4). Disponível em: http://dx.doi.org/10.5020/18061230.2016.p602. 


\section{COMO REFERENCIAR ESTE ARTIGO:}

Knihs M da S, Magalhães ALP, Semeão MCP, Santos RL dos, Lanzoni GM de M, Barra DCC. Ferramenta de avaliação da qualidade: mapeamento de sinais clínicos de morte encefálica. Cogit. Enferm. [Internet]. 2021 [acesso em "colocar data de acesso, dia, mês abreviado e ano"]; 26. Disponível em: http://dx.doi.org/10.5380/ ce.v26i0.75140.

Recebido em: 10/07/2020

Aprovado em: 16/02/2021

Editora associada: Luciana Alcântara Nogueira

Autor Correspondente:

Aline Lima Pestana Magalhães

Universidade Federal de Santa - Florianópolis, SC, Brasil

E-mail: aline.pestana@ufsc.br

Contribuição dos autores:

Contribuições substanciais para a concepção ou desenho do estudo; ou a aquisição, análise ou interpretação de dados do estudo - NSK, ALPM, MCPS

Aprovação da versão final do estudo a ser publicado - NSK, ALPM, MCPS, RLS, GMML, DCCB

ISSN 2176-9133

(c) (i)

Copyright (c) 2021 Este é um artigo em acesso aberto distribuído nos termos da Licença Creative Commons Atribuição, que permite o uso irrestrito, a distribuição e reprodução em qualquer meio desde que o artigo original seja devidamente citado. 